\title{
Assessment of the factorial validity and reliability of the ALSFRS-R: a revision of its measurement model
}

\author{
Leonhard A. Bakker ${ }^{1,2}$ (D) Carin D. Schröder ${ }^{2,3} \cdot$ Michael A. van Es $^{1}$ (D) \\ Paul Westers ${ }^{4}$ Johanna M. A. Visser-Meily ${ }^{2,3} \cdot$ Leonard H. van den Berg ${ }^{1}$ (I)
}

Received: 28 February 2017/Revised: 31 May 2017/ Accepted: 1 June 2017 / Published online: 12 June 2017

(C) The Author(s) 2017. This article is an open access publication

\begin{abstract}
The amyotrophic lateral sclerosis functional rating scale-revised (ALSFRS-R) is a widely used primary outcome measure in amyotrophic lateral sclerosis (ALS) clinical practice and clinical trials. ALSFRS-R items cannot, however, validly be summed to obtain a total score, but constitute domain scores reflecting a profile of disease severity. Currently, there are different measurement models for estimating domain scores. The objective of the present study is, therefore, to derive the measurement model that best fits the data for a valid and uniform estimation of ALSFRS-R domain scores. Data from 1556 patients with ALS were obtained from a population-based register in The Netherlands. A random split of the sample provided a calibration and validation set. Measurement models of the ALSFRS-R were investigated using both exploratory factor analyses and confirmatory factor analyses. The measurement model with a four-factor structure (i.e., bulbar, fine motor, gross motor, and respiratory
\end{abstract}

Electronic supplementary material The online version of this article (doi:10.1007/s00415-017-8538-4) contains supplementary material, which is available to authorized users.

Leonard H. van den Berg

L.H.vandenBerg@umcutrecht.nl

1 Department of Neurology, Brain Centre Rudolf Magnus, University Medical Centre Utrecht, Heidelberglaan 100, 3584 CX Utrecht, The Netherlands

2 Centre of Excellence for Rehabilitation Medicine, Brain Centre Rudolf Magnus, University Medical Centre Utrecht and De Hoogstraat Rehabilitation, Utrecht, The Netherlands

3 Department of Rehabilitation, Physical Therapy Science, and Sports Medicine, Brain Centre Rudolf Magnus, University Medical Centre Utrecht, Utrecht, The Netherlands

4 Julius Center for Health Sciences and Primary Care, University Medical Center Utrecht, Utrecht, The Netherlands function), with correlated factors and cross-loading items on dressing and hygiene and turning in bed and adjusting bed clothes on both motor function scales, provided the best fit to the data in both sets. Correlation between factors ranged from weak to modest, confirming that the ALSFRS$\mathrm{R}$ constitutes a profile of four clinically relevant domain scores rather than a total score that expresses disease severity. The internal consistency of the four domain scores was satisfactory. Our revision of the measurement model may allow for a more adequate estimation of disease severity and disease progression in epidemiological studies and clinical trials.

Keywords Amyotrophic lateral sclerosis - Amyotrophic lateral sclerosis functional rating scale-revised .

Confirmatory factor analysis

\section{Introduction}

Amyotrophic lateral sclerosis (ALS) is a progressive neurodegenerative disorder of the motor neurons for which there is currently no effective treatment. Disease progression in ALS is characterized by loss of physical function in various domains, i.e., the bulbar, fine and gross motor, and respiratory domain. The amyotrophic lateral sclerosis functional rating scale (ALSFRS) [1,2] and its revised version (ALSFRS-R) [3] use this loss of function as a marker for disease severity and disease progression. To date, the ALSFRS-R is the most widely applied rating scale in clinical practice and clinical trials as primary or secondary outcome measure. Moreover, it has been translated into various languages [4-8] and adapted for administration to patients via internet [9], administration to patients 
and caregivers via telephone [10-12], and for self-administration [13].

The ALSFRS-R has demonstrated good criterion-related validity, and the inter-rater, intra-rater, and test-retest reliabilities of the ALSFRS-R are excellent [3, 7, 10, 14]. Recent studies have examined the factorial validity, i.e., the extent to which items measure the intended construct, of the ALSFRS-R using exploratory factor analyses [15], confirmatory factor analyses [15-17] and item response theory analyses [15-17], and have shown that its items do not constitute a total score, a general severity score, but rather a profile of domain scores [15-17]. Hence, the ALSFRS-R domain scores and a consistent strategy to estimate them are of special importance. In the literature, however, there appears to be a divide between those who use a measurement model with a four-factor structure (i.e., bulbar, fine and gross motor, and respiratory) $[3,4,7,17,18]$, as hypothesized by the developers of the ALSFRS-R, and those who use an alternative measurement model with a three-factor structure, which combines fine and gross motor domains into one motor domain $[5,15,16]$. Consequently, there is currently not one distinct strategy for estimating ALSFRS-R domain scores.

The application of various measurement models of the ALSFRS-R could give rise to inconsistent results in the literature. The primary objective of the present study is, therefore, to assess the factorial validity of the ALSFRS-R in a large sample of patients with ALS, to derive a measurement model that describes the data best for a valid and uniform estimation of ALSFRS-R domain scores. Furthermore, the internal consistency of these domain scores will be assessed.

\section{Methods}

\section{Sample}

ALSFRS-R data of patients who fulfilled the diagnostic criteria for possible, probable laboratory-supported, probable, and definite ALS, according to the revised El Escorial criteria [19], were obtained from the population-based register in the Netherlands for the cohort 2006-2015. This register was approved by the UMC Utrecht medical ethics review committee.

To obtain the broadest possible cross section and avoid dependency in the data, only the most recent observation per individual was included in the study. The sample $(n=1556)$ was split randomly into a calibration set (S1) and a validation set (S2).

\section{Amyotrophic lateral sclerosis functional rating scale-revised}

The ALSFRS- $\mathrm{R}$ is a disease-specific 12-item instrument that measures the extent to which patients with ALS are capable of performing functional activities independently [3]. The questionnaire is structured on a 5-point scale ranging from 4 to 0 , where 4 indicates no loss of function and 0 total loss of function. The ALSFRS-R was developed to comprise four scales, each measuring one domain affected by the disease.

\section{Statistical analyses}

Exploratory factor analyses (EFA) of ordered categorical data with orthogonal (Varimax) and oblique (Promax) rotations were performed on the raw data of $\mathrm{S} 1$.

Confirmatory factor analyses (CFA) were first conducted on the data of $\mathrm{S} 1$ and subsequently cross-validated in S2. Given the ordered categorical response format of the ALSFRS-R, CFA should be performed with the weighted least square mean- and variance-adjusted (WLSMV) estimator. However, it is impossible to compare non-nested, i.e., three-factor and four-factor, models using the WLSMV estimator. Using a simulation study, Rhemtulla and colleagues demonstrated that for ordered categorical data with five or more response categories, a robust maximum likelihood estimator, such as the maximum likelihood mean- and variance-adjusted (MLMV) estimator, can be used to obtain acceptable estimates [20], thus facilitating direct comparison of models based on Bayesian Information Criterion (BIC). The different models of the ALSFRS-R were, therefore, examined with both estimators.

Goodness-of-fit was evaluated using the $\chi^{2}$ statistic of exact fit, comparative fit index (CFI), Tucker-Lewis index (TLI), and root mean square error of approximation (RMSEA). For acceptable fit, TLI and CFI should be $>0.90$, and RMSEA $<0.08$. Non-nested models were compared with BIC. Nested models were compared with a $\Delta \chi^{2}$ test.

CFA-based estimation of reliability is considered a more adequate method for calculating scale reliability than the traditionally used coefficient alpha [21]. Therefore, scale reliabilities are estimated using parameter estimates of the optimal CFA model.

Data screening and descriptive statistical analyses were conducted in Rstudio [22]. To assess potential bias due to missing data, both complete case and multiple imputation analyses were performed. Multiple imputation, EFA, and CFA were performed in Mplus Version 7 [23]. 


\section{Competing confirmatory factor analytic (CFA) models of the ALSFRS-R}

The first set of models (1a-1d) to be evaluated expressed the hypothesis that ALSFRS-R items constitute four domains. The first model (1a) specified a measurement model with uncorrelated factors and the second (1b) a less constrained measurement model with correlated factors. Subsequent models were respecified based on modification indices (MIs), which provide the expected drop in $\chi^{2}$ if a parameter is freely estimated, and theoretical knowledge.

1

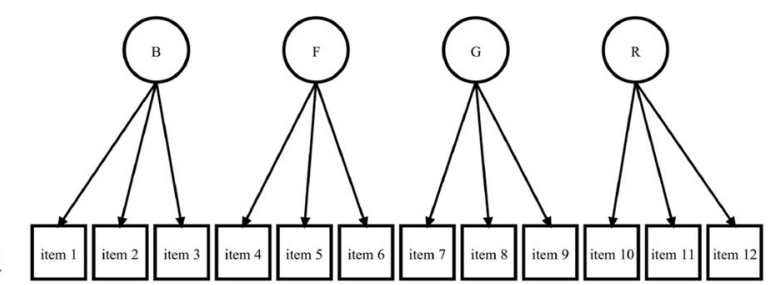

b

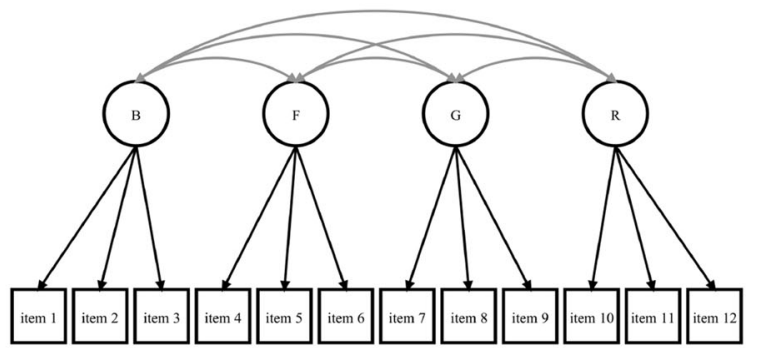

C
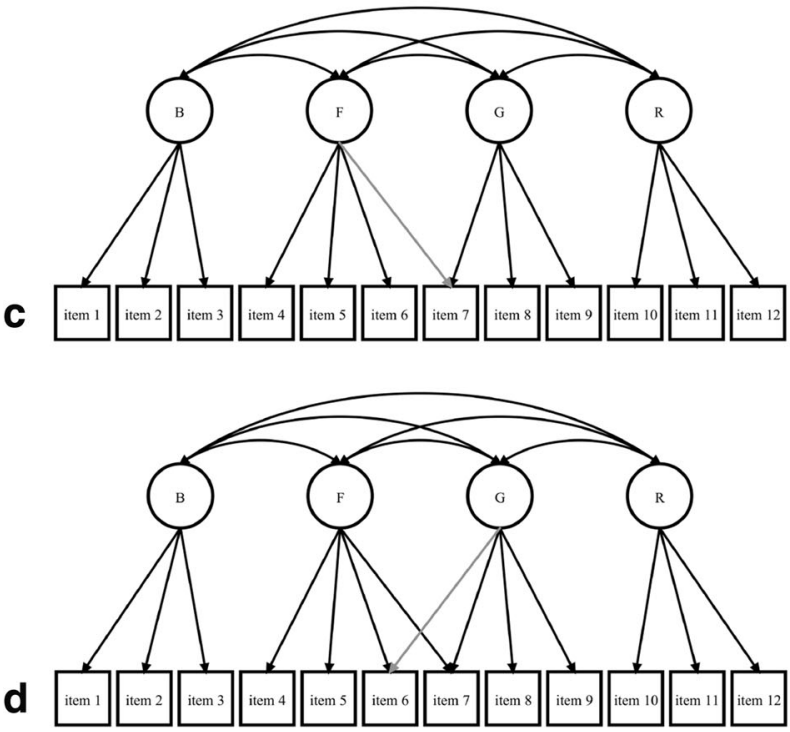

Fig. 1 Path models of measurement models of the ALSFRS-R. Lighter arrows indicate the parameters that were added to the previous models; 1 speech, 2 salivation, 3 swallowing, 4 handwriting, 5 cutting food and handling utensils, 6 dressing and hygiene, 7 turning
The second set of models (2a-2d) expressed the hypothesis that ALSFRS-R items constitute three domains. Again, the first model (2a) specified a measurement model with uncorrelated factors and the second $(2 b)$, a measurement model with correlated factors. Subsequent models were also respecified based on MIs and theoretical knowledge.

The specification of cross-loading items was considered acceptable when an item comprised a combination of functions, while the specification of correlated errors was considered acceptable when respective items had similar content. a

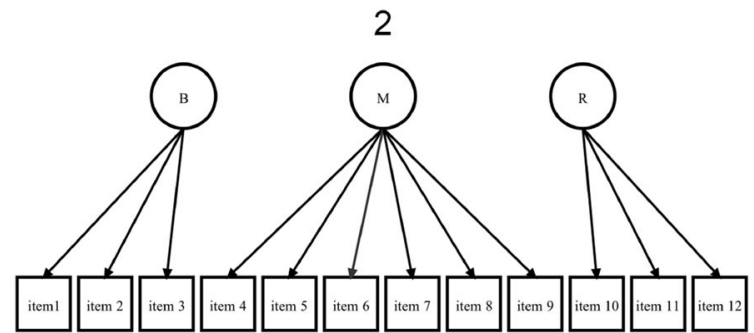

b

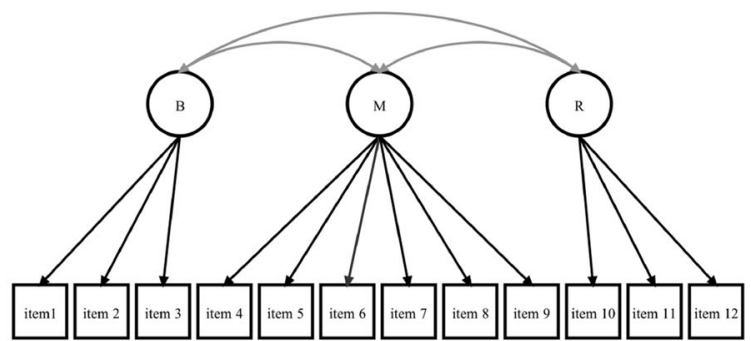

C

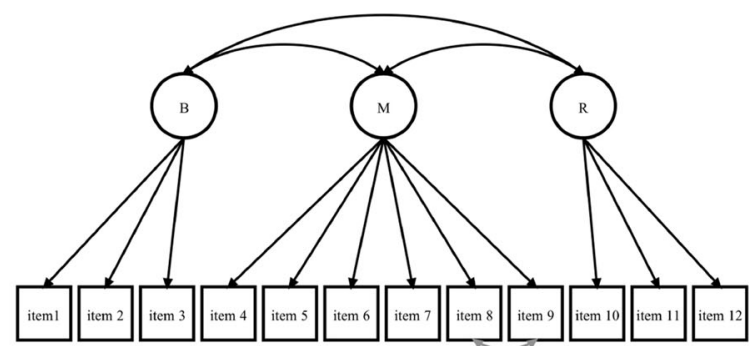

d

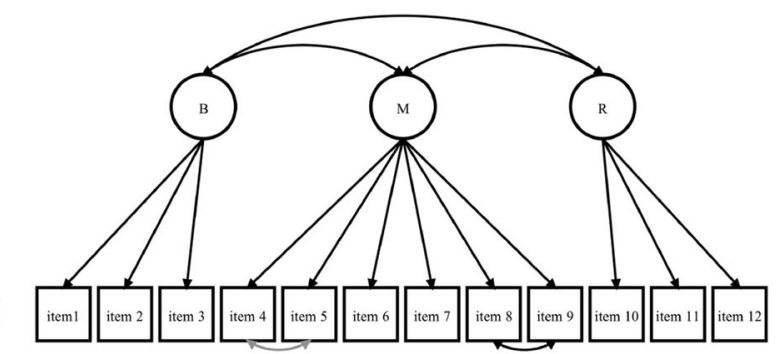

in bed and adjusting bed clothes, 8 walking, 9 climbing stairs, 10 dyspnea, 11 orthopnea, 12 respiratory insufficiency, $B$ bulbar function, $F$ fine motor function, $G$ gross motor function, $M$ motor function, $R$ respiratory function 
Path diagrams of competing models are depicted in Fig. 1. Mplus inputs of both optimal measurement models (1d, 2d) are provided in Online Resources 1 and 2.

\section{Results}

\section{Sample characteristics}

Table 1 presents the demographic and clinical characteristics of both the complete study sample $(N=1556)$ and the two samples that were obtained by a random split of the data $\left(N_{\mathrm{S} 1}=788, N_{\mathrm{S} 2}=788\right)$. As shown in Table 1 , the two samples were comparable.

\section{Exploratory factor analysis (EFA)}

To explore the measurement model of the ALSFRS-R EFA for four- and three-factor solutions were performed.

The EFA of the three-factor solution produced a poor model fit. Both orthogonal and oblique rotations of the three-factor solutions yielded uninterpretable patterns of factor loadings $\left(\chi^{2}=550.20, \quad d f=33, \quad p<0.001\right.$, RMSEA $=0.14$ ).

The EFA of the four-factor solution gave a better model fit $\left(\chi^{2}=73.21, \quad d f=24, \quad p<0.001\right.$, RMSEA $=0.05)$. Both orthogonal and oblique rotations revealed a pattern of factor loadings that could be interpreted as representing bulbar, fine motor, gross motor, and respiratory function. However, the item on dressing and hygiene loaded onto two factors with orthogonal rotation. Furthermore, the item on turning in bed and adjusting bed clothes loaded onto two factors with both orthogonal and oblique rotation. Factor loading patterns of both rotations are presented in Table 2 .

\section{Testing competing confirmatory factor analytic (CFA) models of the ALSFRS-R}

CFA were performed with WLSMV and MLMV estimators. The two analyses produced a similar pattern of results. Furthermore, potential bias due to missing data was assessed with multiple imputation analyses yielding similar results. These are provided in Online Resource 3.

Table 3 shows fit indices of competing measurement models. Four models were tested in each set of models. In measurement models with a four-factor structure, model fit was poor in the initial model (1a), but improved in subsequent models after the specification of correlated factors and cross-loading items on dressing and hygiene and turning in bed and adjusting bed clothes in the optimal model (1d). In measurement models with a three-factor structure, model fit was poor in the initial model (2a), but improved in subsequent models after the specification of correlated factors and correlated errors between items on walking and climbing stairs and writing and cutting food and handling utensils in the optimal model (2d). For both sets of measurement models, all less constrained models had a significant improvement over more constrained models $(p<0.0001)$.
Table 1 Demographic and clinical characteristics of patients

\begin{tabular}{|c|c|c|c|}
\hline & Sample & S1 & $\mathrm{S} 2$ \\
\hline$n$ & 1556 & 778 & 778 \\
\hline \multicolumn{4}{|l|}{ Sex, $n(\%)$} \\
\hline Female & $637(40.9)$ & $321(41.3)$ & $316(40.6)$ \\
\hline Male & $919(59.1)$ & $457(58.7)$ & $462(59.4)$ \\
\hline \multicolumn{4}{|l|}{ Site of onset, $n(\%)$} \\
\hline Bulbar & $511(32.8)$ & $249(32.0)$ & $262(33.7)$ \\
\hline Spinal & $1039(66.8)$ & $528(67.9)$ & $511(65.7)$ \\
\hline Missing & $6(0.4)$ & $1(0.1)$ & $5(0.6)$ \\
\hline \multicolumn{4}{|l|}{ El Escorial diagnosis, $n(\%)$} \\
\hline Definite ALS & $288(18.5)$ & $144(18.5)$ & $144(18.5)$ \\
\hline Probable ALS & $663(42.6)$ & $316(40.6)$ & $347(44.6)$ \\
\hline Probable ALS lab supported & $277(17.8)$ & $150(19.2)$ & $127(16.3)$ \\
\hline Possible ALS & $328(21.1)$ & $168(21.6)$ & $160(20.6)$ \\
\hline Age, mean (sd) & $64.1(11.0)$ & $64.3(11.2)$ & $63.9(10.8)$ \\
\hline Age at onset, mean (sd) & $62.0(11.3)$ & $62.3(11.5)$ & $61.6(11.0)$ \\
\hline ALSFRS-R, mean $(\mathrm{sd})^{\mathrm{a}}$ & $35.1(8.9)$ & $34.9(9.0)$ & $35.3(8.7)$ \\
\hline
\end{tabular}

ALSFRS-R amyotrophic lateral sclerosis functional rating scale-revised, $S 1$ calibration set, $S 2$ validation set

${ }^{\text {a }}$ Raw total score 
Table 2 Factor loadings of exploratory factor analyses

\begin{tabular}{|c|c|c|c|c|c|c|c|c|}
\hline \multirow[t]{2}{*}{ Indicator } & \multicolumn{4}{|c|}{ Orthogonal } & \multicolumn{4}{|c|}{ Oblique } \\
\hline & V1 & V2 & V3 & V4 & P1 & P2 & P3 & P4 \\
\hline Speech & 0.88 & 0.12 & 0.13 & 0.10 & 0.92 & 0.03 & -0.06 & 0.02 \\
\hline Salivation & 0.84 & -0.01 & 0.14 & 0.02 & 0.88 & -0.10 & -0.01 & -0.03 \\
\hline Swallowing & 0.87 & 0.16 & 0.29 & 0.09 & 0.86 & 0.06 & 0.13 & -0.03 \\
\hline Writing & 0.02 & 0.86 & 0.10 & 0.25 & -0.08 & 0.95 & 0.00 & -0.06 \\
\hline Cutting food and handling utensils & 0.15 & 0.89 & 0.11 & 0.25 & 0.05 & 0.98 & -0.02 & -0.07 \\
\hline Dressing and hygiene & 0.09 & 0.76 & 0.12 & 0.55 & -0.01 & 0.70 & -0.04 & 0.35 \\
\hline Turning in bed and adjusting bedclothes & 0.18 & 0.58 & 0.16 & 0.69 & 0.10 & 0.41 & -0.01 & 0.59 \\
\hline Walking & 0.03 & 0.28 & 0.14 & 0.92 & -0.05 & -0.05 & -0.03 & 1.02 \\
\hline Climbing stairs & 0.08 & 0.33 & 0.20 & 0.89 & -0.01 & 0.02 & 0.03 & 0.95 \\
\hline Dyspnea & 0.15 & 0.03 & 0.81 & 0.05 & -0.02 & -0.06 & 0.89 & -0.09 \\
\hline Orthopnea & 0.26 & 0.11 & 0.79 & 0.17 & -0.04 & -0.02 & 0.81 & 0.04 \\
\hline Respiratory insufficiency & 0.16 & 0.28 & 0.46 & 0.29 & 0.03 & 0.18 & 0.42 & 0.18 \\
\hline
\end{tabular}

Bold type indicates factor loadings $>0.40$

$P 1-P 4$ promax rotated loadings, V1-V4 varimax rotated loadings

Table 3 Fit statistics for confirmatory factor analyses

\begin{tabular}{|c|c|c|c|c|c|c|c|c|c|c|c|c|c|}
\hline \multirow[t]{2}{*}{ Estimator } & \multirow[t]{2}{*}{ Model } & \multicolumn{2}{|l|}{$\chi^{2}$} & \multicolumn{2}{|l|}{$d f$} & \multicolumn{2}{|l|}{ CFI } & \multicolumn{2}{|l|}{ TLI } & \multicolumn{2}{|c|}{ RMSEA } & \multicolumn{2}{|l|}{ BIC } \\
\hline & & $\mathrm{S} 1$ & S2 & $\mathrm{S} 1$ & S2 & S1 & S2 & $\mathrm{S} 1$ & S2 & $\mathrm{S} 1$ & $\mathrm{~S} 2$ & $\mathrm{~S} 1$ & $\mathrm{~S} 2$ \\
\hline \multirow[t]{8}{*}{ MLMV } & $1 \mathrm{a}$ & 863.90 & 781.88 & 54 & 54 & 0.76 & 0.73 & 0.70 & 0.73 & 0.14 & 0.13 & 23620 & 23553 \\
\hline & $1 b$ & 444.47 & 400.01 & 48 & 48 & 0.88 & 0.85 & 0.84 & 0.85 & 0.10 & 0.10 & 23022 & 23040 \\
\hline & $1 \mathrm{c}$ & 253.27 & 220.37 & 47 & 47 & 0.94 & 0.95 & 0.91 & 0.93 & 0.08 & 0.07 & 22738 & 22777 \\
\hline & $1 d$ & 152.95 & 134.41 & 46 & 46 & 0.97 & 0.97 & 0.95 & 0.96 & 0.06 & 0.05 & 22594 & 22655 \\
\hline & $2 \mathrm{a}$ & 732.83 & 701.17 & 54 & 54 & 0.80 & 0.80 & 0.75 & 0.76 & 0.13 & 0.13 & 23435 & 23436 \\
\hline & $2 b$ & 610.05 & 600.23 & 51 & 51 & 0.83 & 0.83 & 0.78 & 0.78 & 0.12 & 0.12 & 23240 & 23301 \\
\hline & $2 c$ & 330.32 & 312.85 & 50 & 50 & 0.90 & 0.92 & 0.89 & 0.89 & 0.09 & 0.08 & 22830 & 22888 \\
\hline & $2 d$ & 226.89 & 230.11 & 49 & 49 & 0.93 & 0.95 & 0.93 & 0.93 & 0.07 & 0.07 & 22680 & 22771 \\
\hline \multirow[t]{8}{*}{ WLSMV } & $1 \mathrm{a}$ & 6312.91 & 4671.77 & 54 & 54 & 0.73 & 0.76 & 0.67 & 0.71 & 0.39 & 0.34 & & \\
\hline & $1 b$ & 733.29 & 632.27 & 48 & 48 & 0.97 & 0.97 & 0.96 & 0.96 & 0.14 & 0.13 & & \\
\hline & $1 \mathrm{c}$ & 431.89 & 345.65 & 47 & 47 & 0.98 & 0.99 & 0.98 & 0.98 & 0.10 & 0.09 & & \\
\hline & $1 d$ & 233.00 & 199.75 & 46 & 46 & 0.99 & 0.99 & 0.99 & 0.99 & 0.07 & 0.07 & & \\
\hline & $2 \mathrm{a}$ & 1312.38 & 998.22 & 54 & 54 & 0.95 & 0.95 & 0.93 & 0.94 & 0.18 & 0.15 & & \\
\hline & $2 b$ & 922.86 & 860.71 & 51 & 51 & 0.96 & 0.96 & 0.95 & 0.95 & 0.15 & 0.15 & & \\
\hline & $2 c$ & 531.94 & 458.15 & 50 & 50 & 0.98 & 0.98 & 0.97 & 0.97 & 0.11 & 0.10 & & \\
\hline & $2 d$ & 341.07 & 325.90 & 49 & 49 & 0.99 & 0.99 & 0.98 & 0.98 & 0.09 & 0.09 & & \\
\hline
\end{tabular}

$B I C$ Bayesian information criterion, $C F I$ comparative fit index, $M L M V$ maximum likelihood means and variance, $R M S E A$ root mean square error of approximation, $S 1$ calibration set, $S 2$ validation set, $T L I$ Tucker-Lewis index, WLSMV weighted least squares means and variance

A comparison of BIC values in Table 3 shows that the four-factor model with cross-loading items (1d) has a lower BIC value than the three-factor model with correlated errors (2d), indicating that the former model has a better fit to the data. Models tested in S1 were cross-validated in S2. Furthermore, Table 3 demonstrates that patterns in S2 were similar to patterns in S1.
Table 4 shows fully standardized factor loadings from model 1d in S2. Inspection of the estimates reveals that there is quite some variation between factor loadings, indicating that certain items contribute more to their respective domain score than others. Furthermore, correlations between factors range from weak to modest, indicating that ALSFRS-R subscales do not constitute one overall severity score. 
Table 4 Estimates of the optimal confirmatory factor analytic model

\begin{tabular}{|c|c|c|c|c|}
\hline Domains, items & Reliability & $95 \% \mathrm{CI}$ & $\begin{array}{l}\text { Factor } \\
\text { loadings, } \\
\text { correlations }\end{array}$ & $95 \% \mathrm{CI}$ \\
\hline Bulbar function & 0.87 & $0.85-0.89$ & & \\
\hline Speech & & & 0.89 & $0.87-0.92$ \\
\hline Salivation & & & 0.83 & $0.79-0.86$ \\
\hline Swallowing & & & 0.94 & $0.92-0.96$ \\
\hline Fine motor function & 0.90 & $0.89-0.92$ & & \\
\hline Writing & & & 0.87 & $0.85-0.90$ \\
\hline Cutting food and handling utensils & & & 0.95 & $0.92-0.97$ \\
\hline Dressing and hygiene & & & 0.68 & $0.65-0.73$ \\
\hline Turning in bed and adjusting bedclothes & & & 0.47 & $0.42-0.53$ \\
\hline Gross motor function & 0.89 & $0.88-0.91$ & & \\
\hline Dressing and hygiene & & & 0.38 & $0.33-0.43$ \\
\hline Turning in bed and adjusting bedclothes & & & 0.57 & $0.51-0.62$ \\
\hline Walking & & & 0.93 & $0.91-0.96$ \\
\hline Climbing stairs & & & 0.98 & $0.96-1.00$ \\
\hline Respiratory function & 0.76 & $0.71-0.82$ & & \\
\hline Dyspnea & & & 0.77 & $0.71-0.83$ \\
\hline Orthopnea & & & 0.99 & $0.93-1.07$ \\
\hline Respiratory insufficiency & & & 0.76 & $0.67-0.85$ \\
\hline \multicolumn{5}{|l|}{ Bulbar function } \\
\hline Fine motor function & & & 0.23 & $0.16-0.31$ \\
\hline Gross motor function & & & 0.11 & $0.03-0.20$ \\
\hline Respiratory function & & & 0.43 & $0.35-0.52$ \\
\hline \multicolumn{5}{|l|}{ Fine motor function } \\
\hline Gross motor function & & & 0.53 & $0.47-0.59$ \\
\hline Respiratory function & & & 0.35 & $0.26-0.43$ \\
\hline \multicolumn{5}{|l|}{ Gross motor function } \\
\hline Respiratory function & & & 0.32 & $0.23-0.42$ \\
\hline
\end{tabular}

Estimates were obtained with the WLSMV estimator and standardized (STDYX); italic type indicates cross-loading items

\section{Reliabilities of the ALSFRS-R subscales}

Reliabilities of ALSFRS-R subscales were estimated using CFA-based estimation in S2. Reliability coefficients with 95\% confidence intervals are displayed in Table 4. Inspection of these coefficients shows that all subscales demonstrate acceptable to good internal consistency. The narrowness of these confidence intervals indicates that they can be regarded as providing accurate estimates of the internal consistency.

\section{Discussion}

The primary objective of the present study was to assess the factorial validity of the ALSFRS-R. Our main finding is that the measurement model with a four-factor structure and two cross-loading items provides the best fit to the data. This is in contrast to previous studies that adopted a measurement model with a three-factor structure $[5,15,16,24]$, or a simple four-factor structure, i.e., without cross-loading items [17].

Cross-loading items have been listed in tables of previous reports $[2,3,7,18]$, but it seems their significance was not sufficiently recognized. These cross-loadings are, however, consistent with what clinicians come across in the assessment of ALS: that the items on dressing and hygiene and turning in bed and adjusting bed clothes measure activities that comprise both kinds of motor functioning. Including cross-loading items in the measurement model would, therefore, reflect the clinical reality. The application of our measurement model is, therefore, an adequate approach to assess disease severity in patients with ALS in existing data.

For the application of the ALSFRS-R in future studies, a revision of its item set is justified. Ideally, items that 
comprise more than one question are adapted or deleted from the item set during the development of measurement instruments. A revision of the ALSFRS-R item set could comprise items that are considered important indicators of disease severity by clinicians and patients. An example of a set of candidate items can be found in Wicks and colleagues [18], which was developed to measure disease severity in advanced stages of the disease.

Furthermore, our analyses indicate that measurement models of the ALSFRS-R with correlated factors describe the data significantly better than their equivalent with uncorrelated factors. The correlations between factors do, however, range from weak to modest, corroborating previous reports by Franchignoni and colleagues that the hypothesis that the ALSFRS-R is unidimensional is untenable $[15,16]$. Due to this multidimensionality, ALSFRS-R items cannot validly be summed to obtain a total score that represents disease severity. Consequently, ALSFRS-R items constitute domain scores which reflect a profile of disease severity. Moreover, the application of these domain scores may allow a distinction between different trajectories of disease progression [24]. Our revision of the measurement model of the ALSFRS-R may, therefore, allow for a more adequate assessment of disease severity and disease progression in epidemiological studies and clinical trials.

With regard to reliability, our study supports the finding that all ALSFRS-R subscales demonstrated acceptable to good internal consistency.

Strengths of the present study are the use of both a calibration set and a validation set and the use of modification indices to investigate the measurement model of the ALSFRSR. A weakness is that we only used data of patients administered a Dutch version of the ALSFRS-R. The generalizability of our findings should, therefore, be investigated in a crosscultural study. Furthermore, the present study examined the measurement model in the ALS population. Given the heterogeneity of the disease [25], results might be different in subgroups of the population. Future studies should, therefore, assess measurement invariance of the ALSFRS-R between clinical subgroups of patients with ALS.

Our findings do complement earlier findings that ALSFRS$R$ items constitute a profile of domain scores, rather than a total score representing disease severity. Moreover, results of our study indicate that its measurement model should be revised to reflect the fact that the items on dressing and hygiene and turning in bed and adjusting bed clothes measure activities of daily living, which comprise both fine and gross motor functioning. Our findings may, therefore, allow for a more detailed analysis of disease severity and disease progression. Further studies on the measurement properties of the ALSFRS-R are necessary to expand the evidence on the appropriateness of its application.

\section{Compliance with ethical standards}

Conflicts of interest L.A. Bakker, MSc, Dr. C.D. Schröder, Dr. P. Wester, and Prof. Dr. J.M.A. Visser-Meily declare that they have no conflict of interest. Dr. M.A. van Es reports Grants from The Netherlands Organization for Health Research and Development (Veni scheme), The Thierry Latran Foundation, The Dutch ALS foundation (Stichting ALS Nederland), personal fees from Biogen and Baxalta and serves on the biomedical research advisory panel of the motor neuron disease association (MNDA). Prof. Dr. L.H. van den Berg reports Grants from ALS Foundation Netherlands, Grants from The Netherlands Organization for Health Research and Development (Vici scheme), Grants from The Netherlands Organization for Health Research and Development (SOPHIA, STRENGTH, ALS-CarE project), funded through the EU Joint Programme-Neurodegenerative Disease Research, JPND, personal fees from Baxalta, personal fees from Biogen, personal fees from Cytokinetics, other from Prinses Beatrix SpierFonds, other from Latran Foundation, outside the submitted work.

Ethical standards Data were obtained from the population-based register in the Netherlands for the cohort 2006-2015. All patients gave their informed consent prior to their inclusion in this register, which was approved by the UMC Utrecht medical ethics review committee. The study was performed in accordance with the ethical standards laid down in the 1964 Declaration of Helsinki.

Open Access This article is distributed under the terms of the Creative Commons Attribution 4.0 International License (http://crea tivecommons.org/licenses/by/4.0/), which permits unrestricted use, distribution, and reproduction in any medium, provided you give appropriate credit to the original author(s) and the source, provide a link to the Creative Commons license, and indicate if changes were made.

\section{References}

1. The ALS CNTF treatment study (ACTS) phase I-II Study Group (1996) The Amyotrophic Lateral Sclerosis Functional Rating Scale. Assessment of activities of daily living in patients with amyotrophic lateral sclerosis. Arch Neurol 53:141-147

2. Cedarbaum JM, Stambler N (1997) Performance of the Amyotrophic Lateral Sclerosis Functional Rating Scale (ALSFRS) in multicenter clinical trials. J Neurol Sci 152(Suppl 1):S1-S9. doi:10.1016/S0022-510X(97)00237-2

3. Cedarbaum JM, Stambler N, Malta E et al (1999) The ALSFRS$\mathrm{R}$ : a revised ALS functional rating scale that incorporates assessments of respiratory function. J Neurol Sci 169:13-21. doi:10.1016/S0022-510X(99)00210-5

4. Guedes K, Pereira C, Pavan K et al (2010) Cross-cultural adaptation and validation of ALS functional rating scale-revised in Portuguese language. Arq Neuro-Psiquiatr 68:44-47. doi:10. 1590/S0004-282X2010000100010

5. Campos TS, Rodríguez-Santos F, Esteban J et al (2010) Spanish adaptation of the revised Amyotrophic Lateral Sclerosis Functional Rating Scale (ALSFRS-R). Amyotroph Lateral Scler 11:475-477. doi:10.3109/17482968.2010.489115

6. Ohashi Y, Tashiro K, Itoyama Y et al (2001) Study of functional rating scale for amyotrophic lateral sclerosis: revised ALSFRS (ALSFRS-R) Japanese version. No To Shinkei 53:346-355

7. Abdulla S, Vielhaber S, Körner S et al (2013) Validation of the German version of the extended ALS functional rating scale as a patient-reported outcome measure. J Neurol 260:2242-2255. doi:10.1007/s00415-013-6955-6 
8. Koc F, Balal M, Demir T et al (2016) Adaptation to Turkish and reliability study of the revised Amyotrophic Lateral Sclerosis Functional Rating Scale (ALSFRS-R). Arch Neuropsychiatr 53:229-233. doi:10.5152/npa.2016.11334

9. Maier A, Holm T, Wicks P et al (2012) Online assessment of ALS functional rating scale compares well to in-clinic evaluation: a prospective trial. Amyotroph Lateral Scler 13:210-216. doi:10. 3109/17482968.2011.633268

10. Kaufmann P, Levy G, Montes J et al (2007) Excellent inter-rater, intra-rater, and telephone-administered reliability of the ALSFRS-R in a multicenter clinical trial. Amyotroph Lateral Scler 8:42-46. doi:10.1080/17482960600888156

11. Kasarskis EJ, Dempsey-Hall L, Thompson MM et al (2005) Rating the severity of ALS by caregivers over the telephone using the ALSFRS-R. Amyotroph Lateral Scler Other Motor Neuron Dis 6:50-54. doi:10.1080/14660820510027107

12. Mannino M, Cellura E, Grimaldi G et al (2007) Telephone follow-up for patients with amyotrophic lateral sclerosis. Eur J Neurol 14:79-84. doi:10.1111/j.1468-1331.2006.01559.x

13. Montes J, Levy G, Albert S, Kaufmann P (2006) Development and evaluation of a self-administered version of the ALSFRS-R. Neurology. doi:10.1212/01.wnl.0000238505.22066.fc

14. Miano B, Stoddard GJ, Davis S, Bromberg MB (2004) Interevaluator reliability of the ALS functional rating scale. Amyotroph Lateral Scler Other Motor Neuron Dis 5:235-239. doi:10. 1080/14660820410021302

15. Franchignoni F, Mora G, Giordano A et al (2013) Evidence of multidimensionality in the ALSFRS-R scale: a critical appraisal on its measurement properties using Rasch analysis. J Neurol Neurosurg Psychiatry 84:1340-1345. doi:10.1136/jnnp-2012304701

16. Franchignoni F, Mandrioli J, Giordano A et al (2015) A further Rasch study confirms that ALSFRS-R does not conform to fundamental measurement requirements. Amyotroph Lateral Scler
Frontotemporal Degener 16:331-337. doi:10.3109/21678421. 2015.1026829

17. Bacci ED, Staniewska D, Coyne KS et al (2016) Item response theory analysis of the Amyotrophic Lateral Sclerosis Functional Rating Scale-Revised in the Pooled Resource Open-Access ALS Clinical Trials Database. Amyotrophic Lateral Sclerosis and Frontotemporal Degeneration 17:157-167. doi:10.3109/ 21678421.2015.1095930

18. Wicks P, Massagli MP, Wolf C, Heywood J (2009) Measuring function in advanced ALS: validation of ALSFRS-EX extension items. Eur J Neurol 16:353-359. doi:10.1111/j.1468-1331.2008. 02434.x

19. Brooks BR, Miller RG, Swash M, Munsat TL (2009) El Escorial revisited: revised criteria for the diagnosis of amyotrophic lateral sclerosis. Amyotrophic Lateral Sclerosis and Other Motor Neuron Disorders 1:293-299. doi:10.1080/146608200300079536

20. Rhemtulla M, Brosseau-Liard PÉ, Savalei V (2012) When can categorical variables be treated as continuous? A comparison of robust continuous and categorical SEM estimation methods under suboptimal conditions. Psychol Methods 17:354-373. doi:10. 1037/a0029315

21. Raykov T (2004) Behavioral scale reliability and measurement invariance evaluation using latent variable modeling. Behav Ther 35:299-331. doi:10.1016/S0005-7894(04)80041-8

22. RStudio Team (2014) RStudio: Integrated development for R

23. Muthén BO, Muthén LK, (2015) Mplus user's guide

24. Rooney J, Burke T, Vajda A, et al (2016) What does the ALSFRS-R really measure? A longitudinal and survival analysis of functional dimension subscores in amyotrophic lateral sclerosis. J Neurol Neurosurg Psychiatry jnnp-2016-314661. doi:10. 1136/jnnp-2016-314661

25. Mandrioli J, Biguzzi S, Guidi C et al (2015) Heterogeneity in ALSFRS-R decline and survival: a population-based study in Italy. Neurol Sci 36:2243-2252. doi:10.1007/s10072-015-2343-6 